\title{
Erratum to: Control of flowering time and spike development in cereals: the earliness per se Eps-1 region in wheat, rice, and Brachypodium
}

\author{
Maria E. Faricelli ${ }^{1}$ Miroslav Valárik ${ }^{1,2}$. Jorge Dubcovsky ${ }^{1}$
}

Published online: 22 August 2016

(C) Springer-Verlag Berlin Heidelberg 2016

\section{Erratum to: Funct Integr Genomics (2010) 10:293-306}

DOI 10.1007/s10142-009-0146-7

The colinearity study between wheat, rice, and Brachypodium for the wheat Earliness per se 1 chromosome region performed by Fricelli et al. (2010) found genes MOT1 and FTSH4 completely linked to Eps- $A^{m} 1$. However, one critical recombinant line was incorrectly classified. Additional progeny tests and crosses for this critical line and the identification of 10 new recombination events between MOT1/FTSH4 and $E p s-A^{m} 1$ reported in a more recent study (Alvarez et al. 2016, FIG 16:365-382, doi: 10.1007/s10142-016-0490-3) demonstrated that these two genes are not completely linked to Eps- $A^{m} 1$. Using this additional information $E p s-A^{m} 1$ was found to be completely linked to $E L F 3$, which is proposed as a candidate gene for $E p s-A^{m} l$ in Alvarez et al. (2016). This last reference study should replace the Fricelli et al. (2010) in future studies.

The online version of the original article can be found under doi:10.1007 /s10142-009-0146-7.

Jorge Dubcovsky

jdubcovsky@ucdavis.edu

1 Department of Plant Sciences, University of California, Mail Stop 1, One Shields Avenue, Davis, CA 95616-8780, USA

2 Present address: Laboratory of Molecular Cytogenetics and Cytometry, Institute of Experimental Botany,

Olomouc, Czech Republic 